\title{
Clinical Development of the CDK4/6 Inhibitors Ribociclib and Abemaciclib in Breast Cancer
}

\author{
Romualdo Barroso-Sousa ${ }^{a}$ Geoffrey I. Shapiro ${ }^{b}$ Sara M. Tolaney ${ }^{a, b}$ \\ a Breast Oncology Program, Department of Medical Oncology, Dana-Farber Cancer Institute and Harvard Medical School, \\ Boston, MA, USA; \\ bEarly Drug Development Center, Department of Medical Oncology, Dana-Farber Cancer Institute and Harvard Medical School, \\ Boston, MA, USA
}

\section{Keywords}

Breast cancer · Cyclin-dependent kinase · Palbociclib . Abemaciclib - Ribociclib

\section{Summary}

Clinical and preclinical data support a significant role for inhibitors of the cyclin-dependent kinases (CDKs) 4 and 6 in the treatment of patients with breast cancer. Recently, based on data showing improvement in progression-free survival, the use of palbociclib (Ibrance; Pfizer, Inc.) in combination with endocrine agents was approved to treat patients with hormone receptor-positive advanced disease. Importantly, 2 other CDK4/6 inhibitors, abemaciclib (LY2835219; Lilly) and ribociclib (LEE011; Novartis), are in the late stage of clinical development. In this review, we will focus on clinical data on these 2 new drugs, highlighting their differences compared to palbociclib in terms of single-agent activity, central nervous system penetration, and common adverse events. In addition, we will present the ongoing clinical trials and discuss future directions in the field.

(C) 2016 S. Karger GmbH, Freiburg

\section{Introduction}

The capabilities of sustained chronic proliferation and evasion of growth suppressor signals are both hallmarks of cancer cells [1, 2]. Thus, the understanding of cell cycle control can provide new targets for cancer therapy [3]. Cyclin-dependent kinases (CDKs) 4 and 6 are members of a family of enzymes that govern this biological process. These proteins interact with $\mathrm{D}$-type cyclins, leading to phosphorylation and ultimate inactivation of the retinoblastoma $(\mathrm{Rb})$ tumor suppressor protein. By inactivating $\mathrm{Rb}, \mathrm{CDK} 4$ and CDK6 promote progression from gap $1(\mathrm{G} 1)$ into the DNA synthesis (S) phase of the cell cycle [4].

Several genetic and epigenetic mechanisms have been associated with deregulation of the CDK4/6-D-type cyclin-Rb pathway in different cancers, including (1) loss or inactivating mutation of $\mathrm{Rb}$, (2) amplification of CDK4 or genes encoding D-type cyclins, most notably CCND1, (3) overexpression of D-cyclins in the absence of gene amplification, or (4) loss of function of endogenous CDK4/6 inhibitors of the INK4 family, including p16 ${ }^{\mathrm{INK} 4 \mathrm{~A}}$, which can occur by gene deletion, inactivating mutations, or promoter region hypermethylation [5]. Data from the Cancer Genome Atlas (TCGA) have shown that CCND1 amplification is a common event in breast cancer, and occurs in $29 \%, 58 \%$, and $38 \%$ of luminal A, luminal B, and human epidermal growth factor receptor 2 (HER2)enriched tumors, respectively [6]. In addition, CDK4 gain is found in $25 \%, 24 \%$, and $14 \%$ of luminal A, luminal B, and HER2-enriched tumors, respectively [6]. More importantly, preclinical work has suggested that the activity of antiestrogen drugs on hormone receptor (HR)-positive breast cancer cells may occur through decreased cyclin D1 expression/activity and subsequent impairment in $\mathrm{Rb}$ phosphorylation [7]. Furthermore, another study demonstrated that resistance to endocrine therapy in HR-positive breast tumors may be associated with cyclin D1 overexpression and $\mathrm{Rb}$ phosphorylation [8]. Taken together, these findings suggest that therapies targeting the CDK4/6-D-type cyclin-Rb pathway may have a role in the treatment of breast cancer, particularly in the HR-positive subtype $[9,10]$.

In this context, there is a significant clinical interest in the development of CDK4/6 inhibitors for the treatment of breast cancer. Currently, there is $1 \mathrm{CDK} 4 / 6$ inhibitor that is approved by the Food and Drug Administration (FDA), palbociclib (Ibrance; Pfizer Inc.), and 2 others are in development, abemaciclib (LY2835219;

\section{KARGER}

Fax +497614520714
(๑) 2016 S. Karger GmbH, Freiburg

$1661-3791 / 16 / 0113-0167 \$ 39.50 / 0$
Sara M. Tolaney, MD

Department of Medical Oncology

Dana-Farber Cancer Institute

450 Brookline Avenue, Yawkey 1257, Boston, MA 02215, USA

sara_tolaney@dfci.harvard.edu 
Table 1. Major differences in the CDK4/6 inhibitors palbociclib, abemaciclib, and ribociclib

\begin{tabular}{|c|c|c|c|c|c|c|}
\hline & \multicolumn{2}{|c|}{ Palbociclib } & \multicolumn{2}{|c|}{ Abemaciclib } & \multicolumn{2}{|l|}{ Ribociclib } \\
\hline \multirow[t]{2}{*}{$\mathrm{IC}_{50}$} & \multicolumn{2}{|c|}{ CDK4: 9-11 $\mu \mathrm{M}$} & \multicolumn{2}{|c|}{ CDK4: $2 \mu \mathrm{M}$} & \multicolumn{2}{|c|}{ CDK4: $11 \mu \mathrm{M}$} \\
\hline & \multicolumn{2}{|c|}{ CDK6: $15 \mu \mathrm{M}$} & \multicolumn{2}{|c|}{ CDK6: $5 \mu \mathrm{M}$} & \multicolumn{2}{|c|}{ CDK6: $39 \mu \mathrm{M}$} \\
\hline Dosing & \multicolumn{2}{|c|}{$\begin{array}{l}125 \mathrm{mg} \text { daily } \\
\text { (3 weeks on, } 1 \text { week off) }\end{array}$} & \multicolumn{2}{|c|}{$\begin{array}{l}200 \text { mg twice daily } \\
\text { (continuously) }\end{array}$} & \multicolumn{2}{|c|}{$\begin{array}{l}600 \mathrm{mg} \text { daily } \\
\text { ( } 3 \text { weeks on, } 1 \text { week off) }\end{array}$} \\
\hline ORR in monotherapy, $\%^{\mathrm{a}}$ & \multicolumn{2}{|l|}{6} & \multicolumn{2}{|l|}{17} & \multicolumn{2}{|l|}{3} \\
\hline CNS penetration & \multicolumn{2}{|l|}{ no } & \multicolumn{2}{|l|}{ yes } & \multicolumn{2}{|l|}{ no } \\
\hline Common adverse events, $\%^{\mathrm{a}}$ & all grades & grade $3 / 4$ & all grades & grade $3 / 4$ & all grades & grade $3 / 4$ \\
\hline Neutropenia & 95 & 54 & 88 & 27 & 46 & 29 \\
\hline Thrombocytopenia & 76 & 19 & 42 & 2 & 37 & 10 \\
\hline Fatigue & 68 & 0 & 65 & 13 & 29 & 3 \\
\hline Diarrhea & 16 & 0 & 90 & 20 & 22 & 3 \\
\hline Nausea & 23 & 0 & 65 & 5 & 46 & 2 \\
\hline Vomiting & 5 & 0 & 35 & 2 & 25 & 0 \\
\hline Creatinine increase & NR & NR & 98.5 & 0.8 & NR & NR \\
\hline QTc prolongation & NR & NR & NR & NR & 8 & 0 \\
\hline
\end{tabular}

$\mathrm{CDK}=$ Cyclin-dependent kinase, $\mathrm{CNS}$ = central nervous system, $\mathrm{IC}_{50}$ = half-maximal inhibitory concentration, $\mathrm{HR}$ = hormone receptor, $\mathrm{ORR}=$ objective response rate, $\mathrm{QTc}=$ corrected $\mathrm{QT}$ interval.

${ }^{a}$ The single-agent activity and common adverse events shown in this table are those reported in $[23,29,14]$ for palbociclib, abemaciclib, and ribociclib, respectively.
Lilly) and ribociclib (LEE011; Novartis). Importantly, preclinical data have demonstrated that all 3 drugs inhibit CDK4 and CDK6 with low $\mathrm{IC}_{50}$ values, and each has activity against $\mathrm{Rb}$-proficient human tumor xenograft models [5]. In early 2015, palbociclib received accelerated approval for first-line treatment of postmenopausal women with HR-positive/HER2-negative advanced breast cancer, given in combination with letrozole [11]. The approval was granted based on the results of the randomized, phase 2 PALOMA-1 trial, which showed an improvement in median progression-free survival (PFS) favoring the combination of letrozole plus palbociclib versus letrozole plus placebo (median PFS 20.2 vs. 10.2 months; hazard ratio $(\mathrm{HR})=0.48 ; \mathrm{P}<0.001)$ [12]. Results from the PALOMA-2 study, a phase 3 randomized clinical trial, confirmed these findings [29]. This study showed a significant benefit of the combination of letrozole plus palbociclib in first-line treatment of postmenopausal women with HR-positive/HER2-negative advanced breast cancer: the median PFS was 24.8 vs. 14.5 months, respectively, for patient treated with the palbociclib or placebo $(\mathrm{HR}=0.58 ; \mathrm{P}<0.000001)$. Furthermore, based on data of the PALOMA-3 trial [13], a randomized phase 3 trial evaluating the combination of fulvestrant plus palbociclib versus fulvestrant plus placebo in pre- and postmenopausal patients with HR-positive/ HER2-negative advanced breast cancer, palbociclib received FDA approval in combination with fulvestrant for the treatment of women with HR-positive/HER2-negative advanced breast cancer with disease progression following endocrine therapy. This study showed an improvement in median PFS favoring the arm with palbociclib (median PFS 9.5 vs. 4.6 months; $\mathrm{HR}=0.46 ; \mathrm{P}<0.001$ ). Of note, data from the cohort of pre-/peri-menopausal women included in the PALOMA-3 confirmed the superiority of the combination of palbociclib plus fulvestrant (plus goserelin) vs. fulvestrant (plus goserelin) within this population [30]. In both trials, the most common side effects observed in palbociclibtreated patients were non-febrile neutropenia and fatigue $[12,13]$.

In this review, we will discuss the clinical development of abemaciclib and ribociclib. Importantly, although all 3 drugs acts as CDK4 and CDK6 inhibitors with a high degree of selectivity, they exhibit varying $\mathrm{IC}_{50}$ values against other $\mathrm{CDK}$ family members and utilize different dosing schedules, with some differences in their toxicity profiles (table 1).

\section{Ribociclib}

\section{Monotherapy}

The phase 1 dose escalation trial of ribociclib monotherapy enrolled 128 patients with Rb-positive advanced solid tumors and lymphomas, including 18 patients with breast cancer (NCT01237236). The study established the maximum tolerated dose (MTD) and the recommended phase 2 dose (RP2D) as 900 and $600 \mathrm{mg} /$ day, respectively, on a 21 of 28-day schedule [14]. Among 78 patients evaluable for MTD determination, 10 doselimiting toxicities (DLTs) were observed in cycle 1, including neutropenia $(n=3)$, thrombocytopenia $(n=2)$, mucositis, pulmonary embolism, hyponatremia, increased creatinine level, and asymptomatic QT corrected (QTc) prolongation ( $\mathrm{n}=1$ each). The most common treatment-related adverse events among the 63 patients who received RP2D were neutropenia (all grades $=46 \%$; grade $3 / 4$ $=29 \%$ ), leukopenia (all grades $=48 \%$; grade $3 / 4=21 \%$ ), thrombocytopenia (all grades $=37 \%$; grade $3 / 4=10 \%$ ), anemia (all grades $=30 \%$; grade $3 / 4=3 \%$ ), nausea (all grades $=46 \%$; grade 3 $=2 \%$ ), fatigue (all grades $=29 \%$; grade $3 / 4=3 \%$ ), vomiting (all 
grades $=25 \%$; grade $3=$ none), and diarrhea (all grades $=22 \%$; grade $3 / 4=3 \%$ ). Asymptomatic QTc prolongation (> $450 \mathrm{~ms}$ ) was seen in $8 \%$ of the patients at $600 \mathrm{mg} /$ day (table 1 ).

The agent had a half-life of approximately $36 \mathrm{~h}$. In addition, pharmacodynamic assessment using paired skin biopsies from 40 patients showed reductions of at least 50\% from baseline in Ki67 and phosphorylated $\mathrm{Rb}$, in $55 \%$ and $42 \%$ of the samples, respectively. 3 (2.7\%) of 110 patients evaluable for response had an objective response. In addition, this trial also evaluated a continuous schedule for ribociclib; although final safety and efficacy data are still pending.

\section{Combination with Antiestrogens}

Three large, international, double-blinded, placebo-controlled, phase 3 trials are evaluating the role of adding ribociclib to endocrine therapy in women with HR+/HER2-negative breast cancer.

First, MONALEESA-2 (Mammary ONcology Assessment of LEE011's Efficacy and SAfety-2; NCT01958021) is a phase 3 randomized, double-blind, placebo-controlled, multicenter global registration trial to evaluate the safety and efficacy of ribociclib in combination with letrozole compared to letrozole alone in postmenopausal women with HR-positive/HER2-negative advanced breast cancer, who received no prior therapy for their advanced breast cancer. The trial was conducted at 294 globally distributed clinical trial sites and randomized 668 patients in a 1:1 ratio stratified by the presence of liver and/or lung metastases. Patients received ribociclib $600 \mathrm{mg}$ daily (3 weeks on and 1 week off) or placebo, in combination with letrozole $2.5 \mathrm{mg}$ daily per the approved label. The primary endpoint of the trial was PFS. Secondary endpoints included overall survival (OS), overall response rate (ORR), clinical benefit rate, health-related quality of life, safety, and tolerability. In May 2016, the Independent Data Monitoring Committee recommended stopping the trial early as it met the primary endpoint, significantly extending PFS compared to letrozole alone, at the pre-planned interim analysis. The second phase 3 trial is MONALEESA-7 (NCT02278120), which is currently enrolling premenopausal patients to receive the gonadotropin-releasing hormone agonist goserelin, in combination with a non-steroidal aromatase inhibitor (letrozole or anastrozole), or tamoxifen, with or without ribociclib, also in the first-line setting. The third study is the phase 3 trial MONALEESA-3 (NCT02422615), in which postmenopausal patients with advanced HR-positive/HER2-negative breast cancer are randomized to receive fulvestrant with or without ribociclib in the first- or second-line setting. The primary objective of these trials is also PFS, and secondary endpoints include OS, ORR, and safety (supplemental table 1, www.karger.com/?DOI=447284).

\section{Other Combinations}

Given that the expression of cyclin D1 is in part regulated by the $\mathrm{PI} 3 \mathrm{~K} / \mathrm{Akt} / \mathrm{mTOR}$ pathway $(\mathrm{PI} \mathrm{K}=$ phosphoinositide 3 -kinase, $\mathrm{mTOR}=$ mammalian target of rapamycin) [15] and that over $70 \%$ of breast cancers show activation of this pathway through different mechanisms [16], there is a compelling rationale to investigate the combination of CDK inhibitors and PI3K/mTOR inhibitors. The combination of ribociclib plus the alpha-specific PI3K inhibitor alpelisib (BYL719) has demonstrated synergistic activity in PIK3CAmutant breast cancer cell lines in vitro and in mouse models [17]. The combination was also effective in breast cancer models with intrinsic or acquired resistance to PI3K inhibition [17]. In this context, phase $1 \mathrm{~b} / 2$ studies with ribociclib have been evaluating the safety and efficacy of this drug in triplet combinations with aromatase inhibitors and either everolimus or PI3K inhibitors.

Bardia et al. [18] have presented results of a phase $1 \mathrm{~b} / 2$ study evaluating the safety and efficacy of triplet therapy with ribociclib plus exemestane and everolimus (NCT01857193). In this trial, 70 postmenopausal women with anastrozole- or letrozole-resistant HR-positive/HER2-negative advanced breast cancer received escalating doses of ribociclib once daily, on days 1-21 of 28-day cycles, with everolimus 1-5 $\mathrm{mg}$ daily and exemestane $25 \mathrm{mg}$ daily. The most common grade $3 / 4$ treatment-related adverse events were neutropenia (45.7\%), leukopenia (8.6\%), and thrombocytopenia (5.7\%). DLTs were reported in 6 patients treated with ribociclib $300 \mathrm{mg}$ and everolimus $2.5 \mathrm{mg}$ : increased alanine aminotransferase/aspartate aminotransferase levels (2 patients), febrile neutropenia and hypophosphatemia, oral mucositis, rash and thrombocytopenia, and thrombocytopenia with bleeding (1 patient each). The RP2D was $300 \mathrm{mg} /$ day (3 weeks on/1 week off) $+2.5 \mathrm{mg} /$ day everolimus (continuous) $+25 \mathrm{mg} /$ day exemestane (continuous) with food. Notably, 2.5-mg doses of everolimus in the triplet combination achieved exposure levels broadly equivalent to 5-10 $\mathrm{mg}$ of single-agent everolimus. Among 55 patients evaluable for response, there was $1(1.8 \%)$ complete response (CR), 2 (3.6\%) confirmed and 3 (5.5\%) unconfirmed PRs, and 26 instances (47.3\%) of stable disease (SD). Of note, the median number of prior regimens was 5 , and $18(25.7 \%)$ patients had received prior PI3K/Akt/mTOR or CDK4/6 inhibitors for metastatic disease. The dose expansion part of the study is evaluating both the triplet combination of ribociclib plus everolimus and exemestane at the RP2D and the doublet combination of ribociclib plus exemestane for safety and tolerability.

Another triplet combination study is the 3 -arm phase $1 \mathrm{~b} / 2$ trial that has investigated the combination of ribociclib, letrozole, and alpelisib in patients with HR-positive/HER2-negative advanced breast cancer (NCT01872260) [19]. Patients received a fixed dose of letrozole $(2.5 \mathrm{mg})$ with escalating doses of either oral ribociclib (days 1-21 of 28-day cycles) or apelisb (continuous), or both, in 28 -day cycles. A total of 41 patients received ribociclib plus letrozole (arm 1), 21 patients received alpelisib plus letrozole ( $\operatorname{arm} 2$ ), and 36 patients received ribociclib (300-500 $\mathrm{mg}$ ) plus alpelisib (200-250 mg) plus letrozole. The most common grade 3/4 treatment-related adverse events were nausea (6\%), hyperglycemia (17\%), neutropenia (22\%), and fatigue (11\%). The RP2D was ribociclib $300 \mathrm{mg} /$ day (3 weeks on/1 week off) + alpelisib $200 \mathrm{mg} /$ day (continuous) + letrozole $2.5 \mathrm{mg} /$ day (continuous). Of 27 patients evaluable for response, 2 (7\%) patients had confirmed and 4 (15\%) patients had unconfirmed PRs, while $6(22 \%)$ patients had SD as the best response. The number of prior endocrine regimens for advanced disease was: 0 (14 patients), 1-2 (14 patients), 3-4 (7 patients), and $\geq 5$ ( 1 patient); $33 \%$ of the patients had previously re- 
ceived PI3K/Akt/mTOR inhibitors for metastatic disease. Other ongoing trials are evaluating triplet therapies with ribociclib plus fulvestrant and PI3K inhibitors in the metastatic setting (supplemental table 1,www.karger.com/?DOI=447284).

There are now preclinical data to suggest that CDK4/6 inhibitors may also be effective in HER2-positive breast cancer. Goel et al. [20] showed that cyclin D1/CDK4 is implicated in resistance to anti-HER2 therapy in HER2-positive breast cancer. In addition, CDK4/6 inhibitors were able to resensitize patient-derived xenograft models with acquired resistance to HER2-targeted therapies and delay tumor recurrence in vivo [20]. Currently, there is an ongoing open-label, phase $1 \mathrm{~b} / 2$ clinical trial evaluating ribociclib in combination with trastuzumab ( $\operatorname{arm} \mathrm{A}$ ) or trastuzumab emtansine (T-DM1) (arm B) for patients with HER2-positive locally advanced or metastatic breast cancer (NCT02657343).

\section{Abemaciclib}

\section{Monotherapy}

Patnaik et al. [21] published first data on clinical pharmacology, safety, and efficacy of abemaciclib in humans. They conducted the JPBA trial, an open-label, phase 1, dose escalation study, followed by tumor-specific expansion cohorts. During the dose escalation phase, abemaciclib was given at doses of 50-225 mg daily and 75$275 \mathrm{mg}$ twice daily. The RP2D was found to be $200 \mathrm{mg}$ every $12 \mathrm{~h}$. A dose-proportional pharmacokinetic profile was demonstrated with $t_{1 / 2}$ ranging from 17 to $38 \mathrm{~h}$. Pharmacodynamic assessments were performed in both skin keratinocytes and tumors. Despite the half-life, in skin biopsies taken from patients treated once daily, there was partial reversibility of abemaciclib-induced $\mathrm{Rb}$ phosphorylation and topoisomerase II- $\alpha$ expression in samples obtained prior to the next dose, supporting the use of the twice-daily schedule, which produced evidence of sustained target engagement over the dosing interval. Therefore, this schedule was further investigated in the tumor-specific cohorts.

In contrast to palbociclib and ribociclib, fatigue was the DLT of abemaciclib. Common treatment-related adverse events in the single-agent tumor-specific cohorts $(\mathrm{n}=173)$ included fatigue (all grades $=41 \%$; grade $3=3 \%$ ), diarrhea (all grades $=63 \%$; grade $3=$ $5 \%$ ), nausea (all grades $=45 \%$; grade $3=2 \%$ ), vomiting (all grades $=25 \%$; grade $3=1 \%$ ), and anorexia (all grades $=17 \%$; grade $3=$ none). While diarrhea was common, it was manageable with antidiarrheal agents or dose reduction. Of note, hematologic side effects were less frequent than observed with palbociclib and ribociclib: leukopenia (all grades $=25 \%$; grade $3=10 \%$ ), neutropenia (all grades $=23 \%$; grade $3=9 \%$; grade $4=1 \%$ ), thrombocytopenia (all grades $=23 \%$; grade $3=7 \%$, and anemia (all grades $=20 \%$; grade 3 $=4 \%$ ).

The JPBA study included a monotherapy expansion cohort of 47 women with heavily pretreated (median of 7 prior lines of systemic therapy) metastatic breast cancer. Of note, some patients in this cohort were treated with an alternative starting dose of $150 \mathrm{mg}$ twice daily because of low-grade diarrhea that complicated the 200-mg twice-daily dose level. Fewer dose delays and reductions were noted among patients starting at $150 \mathrm{mg}$ twice daily, although the recommended starting dose of abemaciclib remains $200 \mathrm{mg}$ twice daily.

Radiographic responses occurred exclusively in women with HR-positive disease. Among the 36 patients with HR-positive breast cancer, 11 (31\%) achieved PR. Of the 11 responses in the HR-positive population, 7 were observed among 25 patients (28\%) with HER2-negative disease and 4 occurred among 11 patients (36\%) with HER2-positive disease. Among patients with HR-positive disease, the median duration of response was 13.4 months (95\% confidence interval (CI) 3.7-13.4 months) and the median PFS was 8.8 months (95\% CI 4.2-16.0 months). These data suggest that abemaciclib is able to achieve a high monotherapy response in patients with HR-positive breast cancer.

To better evaluate the safety and efficacy of abemaciclib given as monotherapy, the phase 2 single-arm study MONARCH 1 (NCT02102490) was designed [22]. Women with locally advanced or metastatic HR-positive/HER2-negative breast cancer with disease progression following both antiestrogen therapy and at least 1 , but no more than 2 , lines of chemotherapy in the metastatic setting were eligible. Patients with brain metastasis and/or performance status $>1$ were excluded. A total of 132 patients have been included in this trial and were treated with abemaciclib $200 \mathrm{mg}$ administered orally on a continuous schedule every 12 hours until disease progression. Patients had a median of 2 lines of chemotherapy for advanced disease and approximately $90 \%$ of them had visceral disease. The confirmed ORR was $19.7 \%$, the clinical benefit rate (CR + $\mathrm{PR}+\mathrm{SD} \geq 6$ months) was $42.4 \%$, and median PFS was 6.0 months. The most common treatment-related adverse events were: creatinine increase (all grades $=98.5 \%$; grade $3=0.8 \%$ ), diarrhea (all grades $=90.2 \%$; grade $3=19.7 \%$ ), neutropenia (all grades $=87.7 \%$; grade $3 / 426.9 \%$ ), fatigue (all grades $=65.2 \%$; grade $3=12.9 \%$ ). Because abemaciclib is a competitive inhibitor of efflux transporters of creatinine (OCT2, MATE1, and MATE2-K), cystatin C calculated glomerular fraction rate was performed and it was not raised. Most importantly, abemaciclib-induced diarrhea was manageable with antidiarrheal agents or dose reduction, and discontinuations due to adverse events were infrequent (7.6\%).

Although the agents have not been directly compared within the same trial, the monotherapy response rate appears higher than that seen with palbociclib [23] or ribociclib [14]. The reason for the higher abemaciclib monotherapy response rates compared to the other CDK4/6 inhibitors is not clear, but may be related to greater potency for CDK4 inhibition. Alternatively, because abemaciclib produces less neutropenia, it can be given continuously without breaks (table 1), possibly driving senescence and ultimate tumor regression to a greater degree. Furthermore, the reasons for different toxicity profile may be related to the greater selectivity of this compound for CDK4 than for CDK6. However, pharmacokinetic considerations associated with twice-daily dosing cannot be ruled out as alternative explanations (table 1).

Finally, because preclinical data have shown that abemaciclib crosses the blood-brain barrier and prolongs survival in intracra- 
nial human brain tumor xenografts [24], paired cerebrospinal fluid (CSF) and unbound plasma concentrations of abemaciclib were obtained from 10 patients with glioblastoma in the JPBA trial [21]. The results showed that abemaciclib concentrations detected in the CSF approximate those in plasma. Of note, in agreement with preclinical data, 2 patients with glioblastoma have achieved prolonged PFS, highlighting the potential use of abemaciclib in treating primary or metastatic brain tumors. Currently, the ongoing phase 2 JPBO trial (NCT02308020) is evaluating the efficacy of abemaciclib in patients with HR-positive breast cancer, non-small cell lung cancer, or melanoma with brain metastasis. The primary endpoint is the objective intracranial response rate by the Response Assessment in Neuro-Oncology Brain Metastases (RANO-BM) criteria [25] (supplemental table 2, www.karger.com/?DOI=447284).

\section{Combination with Antiestrogens}

Several studies have explored the combination of abemaciclib with endocrine therapy. The previously discussed JPBA trial also enrolled a cohort of 19 patients with metastatic HR-positive breast cancer who were treated with a combination of abemaciclib 200 mg twice daily plus fulvestrant [21]. All patients had received prior endocrine therapy and had received a median of 4 prior systemic therapies. PRs were observed in 4 patients. The most common treatment-related side effects were similar to those observed in the single-agent cohorts, but grade 3 neutropenia was somewhat higher and was observed in 6 patients (32\%).

The ongoing phase $1 \mathrm{~b}$ study JPBH (NCT02057133) has evaluated the safety, pharmacokinetics, and antitumor activity of abemaciclib combined with a variety of anti-breast cancer therapies [26]. Patients in 6 cohorts received abemaciclib with letrozole 2.5 mg daily (part A), anastrozole 25 mg daily (part B), tamoxifen 20 $\mathrm{mg}$ daily (part C), exemestane $25 \mathrm{mg}$ daily (part D), exemestane 25 mg daily plus everolimus $5 \mathrm{mg}$ daily (part E), or trastuzumab 6-8 $\mathrm{mg} / \mathrm{kg}$ every 21 days (part F). Patients in parts A-E had HR-positive/HER2-negative metastatic breast cancer and those in part $\mathrm{F}$ had HER2-positive metastatic breast cancer. Eligibility included no prior chemotherapy for metastatic disease (parts A-E) and $\geq 1$ chemotherapy regimen for metastatic disease (part F). Preliminary data from the first 65 patients included in parts A-D have been presented and demonstrated that the most common treatmentrelated adverse events were diarrhea (all grades $=95 \%$; grade $3=$ $31 \%$ ), fatigue (all grades $=71 \%$; grade $3=14 \%$ ), nausea (all grades $=62 \%$; grade $3=6 \%$ ), and neutropenia (all grades $=31 \%$; grade $3=$ $17 \%)$. Diarrhea was manageable with antidiarrheal agents or dose reduction. The disease control rate $(C R+P R+S D)$ was $67 \%$ for parts $\mathrm{A}+\mathrm{B}$ (non-steroidal aromatase inhibitors (36 patients)), including 2 confirmed PRs, and 75\% for part C (tamoxifen (16 patients))

Abemaciclib is also being explored in the preoperative setting. A phase 2, open-label, randomized, neoadjuvant trial (neoMONARCH; NCT02441946) is evaluating the biological effects of abemaciclib in combination with anastrozole compared with those of abemaciclib alone and anastrozole alone in tumors of postmenopausal women with HR-positive/HER2-negative breast cancer. The primary endpoint is the change in Ki67 expression from baseline to 2 weeks. The study also explores if prophylactic anti-diarrheal therapy may be beneficial.

Finally, 2 large phase 3, randomized, double-blind, placebocontrolled trials are evaluating the combination of abemaciclib plus antiestrogens, and accrual to both of these studies is complete. In the MONARCH 3 trial (NCT02246621), investigators randomized 450 postmenopausal women with metastatic HR-positive breast cancer in a 2:1 randomization to receive a non-steroidal aromatase inhibitor (letrozole or anastrozole) with or without abemaciclib $150 \mathrm{mg}$ twice daily as first-line therapy for their disease. In the MONARCH 2 (NCT02107703) study, the investigators randomized 550 patients with metastatic $\mathrm{HR}$-positive breast cancer in a 2:1 randomization to receive fulvestrant with or without abemaciclib $150 \mathrm{mg}$ twice daily. For both the MONARCH 3 and MONARCH 2 trials, the primary endpoint is PFS and secondary endpoints include OS and ORR (supplemental table 2, www. karger.com/?DOI=447284).

\section{Other Combinations}

Abemaciclib is also being explored in metastatic HRpositive/HER2-positive breast cancer. The monarcHER trial (NCT02675231) is a randomized phase 2 study that will enroll 225 women who had prior exposure to at least 2 HER2-directed therapies in the context of advanced disease. This trial has 3 arms: abemaciclib plus trastuzumab (arm 1), abemaciclib plus fulvestrant plus trastuzumab (arm 2), and treatment of physician's choice with chemotherapy and trastuzumab (arm 3). The primary endpoint will be PFS and secondary endpoints will include OS and ORR according to the Response Evaluation Criteria in Solid Tumors (RECIST) 1.1 (supplemental table 2, www.karger.com/?DOI=447284).

\section{Future Perspectives}

Many important issues still need to be addressed to optimize the use of CDK4/6 inhibitors in clinical practice. Currently, aside from estrogen receptor expression, there is a lack of predictive biomarkers of response and/or resistance to these agents. The best candidates are components of the CDK4/6-D-type cyclin-Rb pathway; of note, however, in the PALOMA-1 trial, CCND1 amplification and loss of $\mathrm{p} 16^{\mathrm{INK} 4 \mathrm{~A}}$ were not found to define patient populations who derived particular benefit from the combination of letrozole and palbociclib [12]. Ongoing basket trials are designed to address this question, and are recruiting patients with genomic alterations in CCND1, CDKN2A, or CDK4. The SIGNATURE trial (NCT02187783) is an open-label phase 2 trial evaluating the efficacy of ribociclib in patients with different metastatic solid tumors or hematological malignancies. However, among patients with breast cancer, the only eligible patients for this trial are those with triple-negative disease.

Given the success of CDK4/6 inhibitors in breast oncology, another very important question that is raised is whether the combination of CDK inhibitors is effective with other therapeutic mo- 
dalities, including chemotherapy, radiotherapy, and immune checkpoint inhibitors. There are concerns about tolerability based on possible overlapping adverse events, including neutropenia, fatigue, and diarrhea. Also critical is whether those combinations will translate into increased benefit for patients. Because cytotoxic chemotherapy and radiotherapy work by killing cells that are cycling, there are concerns that concomitant use of CDK4/6 inhibitors may antagonize these treatment modalities. However, sequential schedules may be of interest; in fact, Clark et al. [27] have recently presented data from a phase $1 \mathrm{~b}$ trial evaluating sequential taxol followed by palbociclib in the breast cancer population. The trial has shown that these drugs can be safely combined with an intermittent dosing schedule. In addition, the efficacy data was promising even in patients previously exposed to a taxane. Furthermore, because previous data showed that $\mathrm{T}$ cell expansion and differentiation are dependent on the cyclin D3/CDK6 function, CDK4/6 inhibitors may be detrimental to the immune microenvironment, so that combinations with immune checkpoint inhibitors, such as monoclonal antibodies against programmed cell death protein 1 (PD-1) or its ligand, PD-L1, may not be synergistic.

Another issue of active research is the role of CDK4/6 inhibitors in triple-negative breast cancer (TNBC). Recently, Asghar et al. [28] have shown that luminal-androgen receptor (LAR) cell lines represent a subgroup of TNBC that may benefit from CDK4/6 inhibition. The sensitivity to these inhibitors correlated with expression of the androgen receptor, and the absence/or low levels of cyclin E1 [28]. Thus, there may be a role for CDK4/6 inhibitors in a subset of patients with androgen receptor-positive TNBC. Finally, there are ongoing clinical trials evaluating the role of continuing CDK4/6 inhibition after progression on CDK4/6 inhibitors (NCT02732119, NCT02632045).

\section{Conclusions}

Selective CDK4/6 inhibitors represent an important therapeutic advance in breast oncology. Besides the clinical success of palbociclib, 2 other drugs, i.e. abemaciclib and ribociclib, are in the late stage of clinical development. The results of large phase 3 trials evaluating these agents in combination with endocrine therapy are expected within the next year. Abemaciclib has a different toxicity profile, and data suggest that it has both a high monotherapy response rate and significant central nervous system penetration. In addition, triplet combinations of hormonal therapy with CDK4/6 inhibitors and inhibitors of the PI3K/Akt/mTOR pathway are ongoing. Further work is needed to determine if continuation of CDK4/6 inhibition beyond progression has any benefit, and studies are planned to address this question.

\section{Online Supplemental Tables}

Supplemental Table 1. Ongoing clinical trials evaluating ribociclib in patients with breast cancer

Supplemental Table 2. Ongoing clinical trials evaluating abemaciclib in monotherapy or in combination with other agents in patients with breast cancer

To access the supplemental tables, please refer to www.karger.com/? $D O I=447284$.

\section{Disclosure Statement}

Sara Tolaney and Geoffrey Shapiro both have received research funding from Lilly, Novartis, and Pfizer.

\section{References}

1 Hanahan D, Weinberg RA: The hallmarks of cancer. Cell 2000;100:57-70.

2 Hanahan D, Weinberg RA: Hallmarks of cancer: the next generation. Cell 2011;144:646-674.

3 Sherr CJ, Beach D, Shapiro GI: Targeting CDK4 and CDK6: from discovery to therapy. Cancer Discov 2016; 6:353-367.

4 Weinberg RA: The retinoblastoma protein and cell cycle control. Cell 1995;81:323-330.

5 Asghar U, Witkiewicz AK, Turner NC, Knudsen ES The history and future of targeting cyclin-dependent kinases in cancer therapy. Nat Rev Drug Discov 2015; 14:130-146.

6 Cancer Genome Atlas Network: Comprehensive molecular portraits of human breast tumours. Nature 2012;490:61-70.

7 Watts CK, Brady A, Sarcevic B, deFazio A, Musgrove EA, Sutherland RL: Antiestrogen inhibition of cell cycle progression in breast cancer cells is associated with inhibition of cyclin-dependent kinase activity and decreased retinoblastoma protein phosphorylation. Mol Endocrinol 1995;9:1804-1813.

8 Thangavel C, Dean JL, Ertel A, Knudsen KE, Aldaz $\mathrm{CM}$, Witkiewicz AK, et al.: Therapeutically activating RB: reestablishing cell cycle control in endocrine therapy-resistant breast cancer. Endocr Relat Cancer 2011; 18:333-345.
Mayer EL: Targeting breast cancer with CDK inhibitors. Curr Oncol Rep 2015;17:443.

10 Murphy CG, Dickler MN: The role of CDK4/6 inhibition in breast cancer. Oncologist 2015;20:483-490.

11 Beaver JA, Amiri-Kordestani L, Charlab R, Chen W, Palmby T, Tilley A, et al.: FDA approval: palbociclib for the treatment of postmenopausal patients with estrogen receptor-positive, HER2-negative metastatic breast cancer. Clin Cancer Res 2015;21:4760-4766.

12 Finn RS, Crown JP, Lang I, Boer K, Bondarenko IM, Kulyk SO, et al.: The cyclin-dependent kinase 4/6 inhibitor palbociclib in combination with letrozole versus letrozole alone as first-line treatment of oestrogen receptor-positive, HER2-negative, advanced breast cancer (PALOMA-1/TRIO-18): a randomised phase 2 study. Lancet Oncol 2015;16:25-35.

13 Cristofanilli M, Turner NC, Bondarenko I, Ro J, Im SA, Masuda N, et al.: Fulvestrant plus palbociclib versus fulvestrant plus placebo for treatment of hormonereceptor-positive, HER2-negative metastatic breast cancer that progressed on previous endocrine therapy (PALOMA-3): final analysis of the multicentre, double-blind, phase 3 randomised controlled trial. Lancet Oncol 2016, doi: 10.1016/S1470-2045(15)00613-0 [Epub ahead of print].
14 Infante JR, Shapiro G, Witteveen P, Gerecitano JF, Ribrag V, Chugh R, et al.: A phase I study of the singleagent CDK4/6 inhibitor LEE011 in pts with advanced solid tumors and lymphomas. ASCO Meeting Abstracts 2014;32(15 suppl):abstr 2528.

15 Sherr CJ: Cancer cell cycles. Science 1996;274:16721677

16 Garcia-Martinez JM, Alessi DR: mTOR complex 2 (mTORC2) controls hydrophobic motif phosphorylation and activation of serum- and glucocorticoidinduced protein kinase 1 (SGK1). Biochem J 2008;416: 375-385.

17 Vora SR, Juric D, Kim N, Mino-Kenudson M, Huynh T, Costa C, et al.: CDK 4/6 inhibitors sensitize PIK3CA mutant breast cancer to PI3K inhibitors. Cancer Cell 2014:26:136-149.

18 Bardia A, Modi S, Oliveira M, Campone M, Ma B, Dirix L, et al.: Triplet therapy with ribociclib, everolimus, and exemestane in women with HR+/HER2advanced breast cancer. Cancer Res 2016;76(4 suppl): abstr P6-13-01.

19 Juric D, Ismail-Khan R, Campone M, García-Estévez L, Becerra C, De Boer R, et al.: Phase Ib/II study of ribociclib and alpelisib and letrozole in ER+, HER2- breast cancer: safety, preliminary efficacy and molecular analysis. Cancer Res 2016;76(4 suppl):abstr P3-14-01. 
20 Goel S, Wang Q, Watt AC, Tolaney SM, Dillon DA, Li $\mathrm{W}$, et al.: Overcoming therapeutic resistance in HER2 positive breast cancers with CDK4/6 inhibitors. Cancer Cell 2016;29:255-269.

21 Patnaik A, Rosen LS, Tolaney SM, et al.: Efficacy and safety of abemaciclib, an inhibitor of CDK4 and CDK6, for patients with breast cancer, non-small cell lung cancer, and other solid tumors. Cancer Discov 2016; doi: 10.1158/2159-8290.CD-16-0095.

22 Dickler MN, Tolaney SM, Rugo HS, et al.: MON ARCH1: Results from a phase II study of abemaciclib, a CDK4 and CDK6 inhibitor, as monotherapy, in patients with HR+/HER2- breast cancer, after chemotherapy for advanced disease. J Clin Oncol 2016;34: (suppl; abstr 510).

23 DeMichele A, Clark AS, Tan KS, Heitjan DF, Gramlich K, Gallagher M, et al.: CDK 4/6 inhibitor palbociclib (PD0332991) in Rb+ advanced breast cancer: phase II activity, safety, and predictive biomarker assessment. Clin Cancer Res 2015;21:995-1001.
24 Raub TJ, Wishart GN, Kulanthaivel P, Staton BA, Ajamie RT, Sawada GA, et al.: Brain exposure of two selective dual CDK4 and CDK6 inhibitors and the antitumor activity of CDK4 and CDK6 inhibition in combination with temozolomide in an intracranial glioblastoma xenograft. Drug Metab Dispos 2015;43: 1360-1371.

25 Lin NU, Lee EQ, Aoyama H, Barani IJ, Barboriak DP, Baumert BG, et al.: Response assessment criteria for brain metastases: proposal from the RANO group. Lancet Oncol 2015;16:e270-e278.

26 Tolaney SM, Beeram M, Beck JT, Conlin AK, Dees EC, Dickler MN, et al.: A phase Ib study of abemaciclib with therapies for metastatic breast cancer. ASCO Meeting Abstracts 2015;33(15 suppl):abstr 522.

27 Clark A, O’Dwyer P, Troxel A, Lal P, Feldman M, Gallagher M, et al.: Palbociclib and paclitaxel on an alternating schedule for advanced breast cancer: results of a phase Ib trial. Cancer Res 2016;76(4 suppl):abstr P6$13-08$.
28 Asghar U, Herrera-Abreu MT, Cutts R, Babina I, Pearson A, Turner NC: Identification of subtypes of triple negative breast cancer (TNBC) that are sensitive to CDK4/6 inhibition. ASCO Meeting Abstracts 2015; 33(15 suppl):abstr 11098.

29 Finn RS, Martin M, Rugo HS, et al.: PALOMA-2: Primary results from a phase III trial of palbociclib (P) with letrozole (L) compared with letrozole alone in postmenopausal women with ER+/HER2- advanced breast cancer (ABC). J Clin Oncol 2016;34:(suppl; abstr 507).

30 Loibl S, Turner NC, Ro J, et al.: Palbociclib (PAL) in combination with fulvestrant $(\mathrm{F})$ in pre-/peri-menopausal (PreM) women with metastatic breast cancer $(\mathrm{MBC})$ and prior progression on endocrine therapy results from Paloma-3. J Clin Oncol 2016;34:(suppl; abstr 524). 